\title{
Anatomical Labeling of the Anterior Circulation of the Circle of Willis Using Maximum a Posteriori Classification
}

\author{
Hrvoje Bogunović, José María Pozo, Rubén Cárdenes, and Alejandro F. Frangi \\ Center for Computational Imaging \& Simulation Technologies in Biomedicine, \\ (CISTIB) - Universitat Pompeu Fabra (UPF) and CIBER-BBN, Barcelona, Spain
}

\begin{abstract}
Automated anatomical labeling of the arteries forming the Circle of Willis is of great interest as facilitates inter-subject comparison required to discover geometric risk factors for the development of vascular pathologies. In this paper, we present a method for anatomical labeling of vessels forming anterior part of the Circle of Willis by detecting the five main vessel bifurcations. The method is first trained on a set of pre-labeled examples, where it learns local bifurcation features as well as global variation in the anatomy of the extracted vascular trees. Then the labeling of the target vascular tree is formulated as maximum a posteriori solution where the classifications of individual bifurcations are regularized by the prior learned knowledge of the tree they span. The method was evaluated by cross-validation on 30 subjects, which showed the vascular trees were correctly anatomically labeled in $90 \%$ of cases. The proposed method can naturally handle anatomical variations and is shown to be suitable for labeling arterial segments of Circle of Willis.
\end{abstract}

\section{Introduction}

The Circle of Willis is a ring of cerebral vessels that connects the two halves of the anterior circulation with the posterior one and its bifurcations are a common site of aneurysm formation (pathological dilations of vessels) [1]. Analyzing the statistical variation of characteristics of these vessels and bifurcations is of great interest as it can lead to the identification of geometric risk factors for the onset of vascular pathologies. Two important operations required for comparing and registering the vasculature between subjects are landmark matching and anatomical labeling. These are tedious and time consuming tasks to be performed manually. Thus, automating them becomes crucial for processing large number of cases.

In this work, we present a method for robust classification of bifurcations in a vascular tree, which we applied to the task of anatomically labeling the anterior part of the Circle of Willis. The anterior part is formed by the three main vessels: internal carotid artery (ICA), middle cerebral artery (MCA) and anterior cerebral artery (ACA). Statistically, 80\% of the aneurysms occur along these vessels [1]. In particular, we are interested in anatomical labeling of the following five bifurcations and the vessels connecting them. The bifurcations of ICA

G. Fichtinger, A. Martel, and T. Peters (Eds.): MICCAI 2011, Part III, LNCS 6893, pp. 330-337, 2011. (C) Springer-Verlag Berlin Heidelberg 2011 


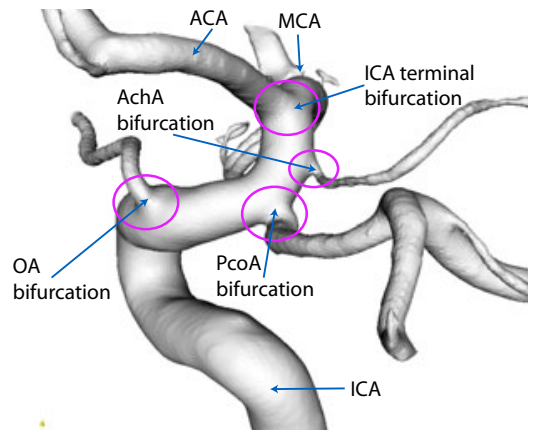

(a)

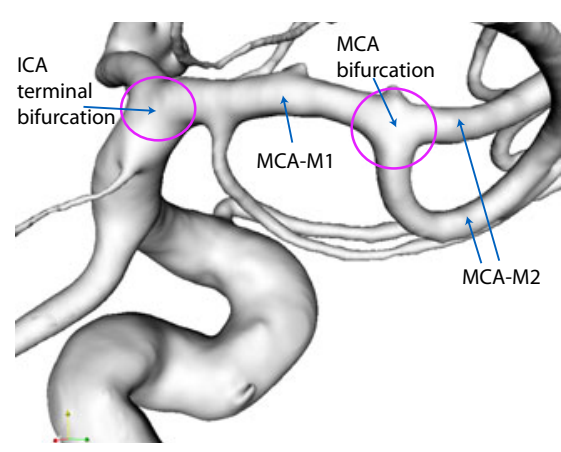

(b)

Fig. 1. The arteries of the Circle of Willis and the bifurcations of interest on: (a) internal carotid artery (ICA), (b) middle cerebral artery (MCA)

with ophthalmic artery (OA), posterior communicating artery (PcoA), anterior choroidal artery (AchA) and the terminal ICA bifurcation, plus the principal bifurcation of MCA, which divides the MCA-M1 and MCA-M2 segments (Fig. 1). To that set of bifurcations, throughout the text, we will refer to as bifurcations of interest (BoI).

The state of the art for anatomical labeling of vasculature is, in general, sparse. The seminal work was done by Tschirren et al. 2] for anatomical labeling of airway trees that relies on branchpoint matching to a prelabeled tree that represents population average. Mori et al. in a series of works 34 presented a knowledgebased framework for anatomical labeling of bronchial branches based on machine learning and combination optimization. However, their method uses a fixed set of topological constraints and does not seem to be robust to large anatomical variations of the reference tree topology. Airway and bronchial trees are characterized by many similar bifurcations connected by short straight branches. This makes the methods design for them difficult to apply to the task of labeling cerebral vessels where the vessels are in general longer and more curved and the bifurcations more complex. Recently, Mori et al. [5] tuned their approach to a specific task of labeling abdominal arteries but even the authors themselves stated that the application to other organs would be challenging. Bogunović et al. [6] showed that the ICA terminal bifurcation can be successfully classified using support vector machine (SVM) but only a single bifurcation is detected and no tree properties are considered.

The rest of the paper is organized as follows. In section 2, we present the workflow of the method, starting from angiographic 3D image, and formulate the classification of bifurcations in a maximum a posteriori framework. Evaluation of the method on a set of 30 images is presented in section 3 . Finally, section 4 discusses the advantages and limitations of the proposed method and concludes the paper. 


\section{Methods}

\subsection{Vascular Tree Extraction and Characterization}

Segmentations of the cerebral vasculature from 3D images were performed in a fully automated way with a geometric deformable model called Geodesic Active Regions (GAR) [7. From the segmented vasculature, fast topological thinning based on collapsing fronts using [8] was applied to obtain a rough estimate of the underlying topology of the vessel tree. Due to image acquisition noise and inaccuracies in vessel segmentation, touching vessels effect can appear, thus causing the extracted topology to form a connected graph containing cycles and not a tree. However, the graph's end-points do correspond to the root and the terminal leafs of the vascular tree. Root was taken to be the end-point with the maximal associated radius at the lowest axial plane which, in our images, corresponded to the ICA entering the field of view. Then, the set of accurate centerlines is obtained by backtracking along the minimal cost path from the end-points toward the root using [9], which is available within the open-source library VMTK [10. These centerlines now form a rooted tree with the edges directed away from the root in accordance with the blood flow (Fig. 2 (a)].

Bifurcation Feature Vector. Each bifurcation of the tree is characterized using the method of Antiga [11, available in VMTK, which relies on objective criteria for defining the origin of a bifurcation and bifurcation vectors of the parent branch and the two daughter branches. The two daughter branches are differentiated by their radius: Larger daughter branch and smaller daughter branch. We chose the following collection of features to quantify the geometry of a bifurcation (Fig. 2 (b)), which form a 21 dimensional, scale invariant, bifurcation feature vector:

- Sagittal, axial and coronal-components of the normal vector of the bifurcation plane (3).

- Sagittal, axial and coronal-components of the three bifurcation vectors (9).

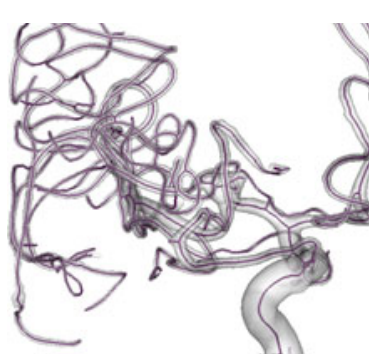

(a)

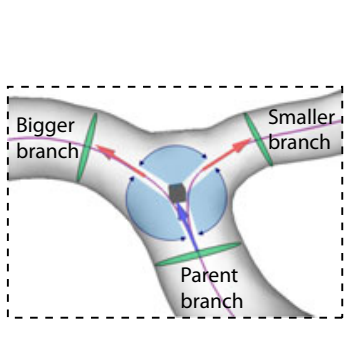

(b)

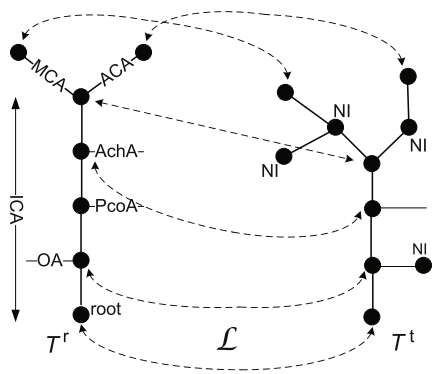

(c)

Fig. 2. Elements of the method's workflow: (a) Centerline extraction. (b) Bifurcation features. (c) Example of a labeling $\mathcal{L}$ (dotted arrows) of a target tree $T^{\mathrm{t}}$ (right) based on a reference tree $T^{\mathrm{r}}$ with the bifurcations of interest (left). 
- Angles between each pair of bifurcation vectors (3).

- Ratios of mean vessel radii between each pair of bifurcation branches (3).

- Ratios of mean vessel radii between each bifurcation branch and the root branch (ICA) of the tree (3).

Tree Features. To characterize the extracted tree, to each pair of bifurcations we associate an inheritance relationship: ancestor-offspring or sibling. In addition, a probability is assigned that a particular bifurcation is present in the tree. In general, one could easily also include features of the vessels connecting the pair of bifurcations, like their mean length, curvature, torsion or tortuosity.

\subsection{Bifurcation Classification}

Extracted vascular tree can be considered as Attributed Relational Tree (ART) which has attributes in a form of feature vectors associated to its vertexes and edges. ART is defined as

Definition 1. Rooted Attributed Relational Tree is a quadruple $T=(V, E, A, r)$, where $V$ is the vertex set, $E$ is the edge set, $r$ is root and $A$ is the attribute set that contains unary attribute $a_{i}$ attaching to each vertex $v_{i} \in V$, and a binary attribute $a_{i, j}$ attaching to each edge $e_{k}=\left(n_{i}, n_{j}\right) \in E$.

The classification of bifurcations is based on the availability of a reference ART $T^{\mathrm{r}}$, created from a representative sample of a population, having BoI as its vertices $V^{\mathrm{r}}=\left\{v_{i}^{\mathrm{r}}: 1 \leq i \leq M\right\}$ together with an entire set of sample bifurcation feature vectors as $\left\{a_{i}^{\mathrm{r}}\right\}$, while edge features $\left\{a_{i, j}^{\mathrm{r}}\right\}$ are currently not used. Such a reference ART is normally created from a training set of trees. The target ART $T^{\mathrm{t}}$ corresponds to the extracted vascular tree, having its bifurcations as vertices $V^{\mathrm{t}}=\left\{v_{j}^{\mathrm{t}}: 1 \leq j \leq N\right\}$ and the bifurcation feature vectors as $\left\{a_{j}^{\mathrm{t}}\right\}$. Then, on the target tree we define a labeling process $\mathcal{L}: V^{\mathrm{t}} \rightarrow V^{\mathrm{r}} \cup\{\mathrm{NI}\}$, where the label NI represents a bifurcation which is not of interest and is not present in the reference ART (Fig. [2](c)).

We are interested in estimating the probability $P\left(\mathcal{L} \mid T^{\mathrm{t}}, T^{\mathrm{r}}\right)$ of $\mathcal{L}$ being correct, and finding the mode of this posterior distribution. Thus, the problem can be formulated as finding a labeling $\mathcal{L}^{*}$ that satisfies the maximum a posteriori (MAP) solution

$$
\begin{aligned}
P\left(\mathcal{L}^{*} \mid T^{\mathrm{t}}, T^{\mathrm{r}}\right) & >P\left(\mathcal{L} \mid T^{\mathrm{t}}, T^{\mathrm{r}}\right) \quad \forall \mathcal{L} \neq \mathcal{L}^{*}, \\
P\left(\mathcal{L} \mid T^{\mathrm{t}}, T^{\mathrm{r}}\right) & \propto P\left(T^{\mathrm{t}} \mid \mathcal{L}, T^{\mathrm{r}}\right) P\left(\mathcal{L} \mid T^{\mathrm{r}}\right),
\end{aligned}
$$

where the constant denominator term in Eq. 2 has been left out for brevity.

The prior term $P\left(\mathcal{L} \mid T^{\mathrm{r}}\right)$ presents our knowledge-based expectations about the topology and tree properties of the labeled target tree and regularizes the classification based on bifurcation features. The likelihood term, assuming that the feature vectors of each bifurcation $\left\{a_{i}\right\}$ are statistically independent from each other, can be written as

$$
P\left(T^{\mathrm{t}} \mid \mathcal{L}, T^{\mathrm{r}}\right)=\prod_{i=1}^{N} P\left(a_{i}^{\mathrm{t}} \mid \mathcal{L}\left(v_{i}^{\mathrm{t}}\right), T^{\mathrm{r}}\right) .
$$


Likelihood Estimation. To estimate $P\left(a_{i}^{\mathrm{t}} \mid \mathcal{L}\left(v_{i}^{\mathrm{t}}\right)\right)$ i.e. a likelihood that a bifurcation with known label $\mathcal{L}\left(v_{i}^{\mathrm{t}}\right)$ has the feature vector $a_{i}^{\mathrm{t}}$, we employed the SVM with probability estimates available in LIBSVM open-source library [12]. A non-linear model based on radial basis function (RBF) kernel was used. For the multi-class problem, several binary SVM were created in a one-against-one strategy where binary SVMs, for all pairs of classes, were trained on the data and the results for each pair accumulate to yield the final probability distribution.

MAP Estimation. Unfortunately MAP estimation is extremely computationally expensive. Testing for all possible labelings $\mathcal{L}$ is not feasible. However, the prior term $P\left(\mathcal{L} \mid T^{\mathrm{r}}\right)$ is positive only for a small subset of all combinations i.e. only for those for which the topology corresponds to the one of the reference tree $T^{\mathrm{r}}$. This happens when $\mathcal{L}$ is an isomorphism from a subgraph of $T^{\mathrm{t}}$ to a subdivision of $T^{\mathrm{r}}$.

A standard algorithm to find all isomorphic mappings between two graphs (trees) is based on building their association graph and then finding all maximal cliques of such undirected graph. Association graph $G=\left(V^{\mathrm{a}}, E^{\mathrm{a}}\right)$ is built from $T^{\mathrm{r}}$ and $T^{\mathrm{t}}$, where nodes are denoted with a pair of indices $V^{\mathrm{a}}=\left\{v^{\mathrm{a}} \equiv\left(v_{i}^{\mathrm{r}}, v_{j}^{\mathrm{t}}\right)\right\} \equiv$ $V^{r} \times V^{t}$, with the following rule. Edge $\left(v_{i, j}^{\mathrm{a}}, v_{k, l}^{\mathrm{a}}\right)$ is created only if $v_{i}^{\mathrm{r}}$ and $v_{k}^{\mathrm{r}}$ have the same relationship (ancestor-offspring or sibling) as do $v_{j}^{\mathrm{t}}$ and $v_{l}^{\mathrm{t}}$, for $i \neq k$ and $j \neq l$. We have now extended the above rules by adding the label NI to $V^{\mathrm{t}}$, hence $V^{\mathrm{a}}=V^{\mathrm{r}} \times\left(V^{\mathrm{t}} \cup\{\mathrm{NI}\}\right)$ with the special rule that NI is in all relationships with any other vertex of $V^{\text {a }}$ including itself.

Finding maximal cliques (in our case they will always be of size N) in an undirected graph is in general NP-complete problem and we used Bron-Kerbosch algorithm [13, that tries to reduce the size of search space, to find the maximal cliques.

\section{Results}

We evaluated the proposed methodology on a dataset consisting of images from 30 subjects, acquired with 3D rotational angiography (3DRA), where contrast injection was carried out to enhance the vessels comprising the anterior cerebral circulation of either left or right hemisphere. Images were reconstructed with a $256^{3}$ matrix having a voxel size of $0.29 \times 0.29 \times 0.29(\mathrm{~mm})$.

All images were successfully segmented and had their centerlines and tree topology extracted. The obtained trees were containing bifurcations and trifurcations. Since two close bifurcations are sometimes reconstructed as a trifurcation, we decided to always treat trifurcations as two bifurcations sharing the same parent branch and the larger daughter branch.

The success of bifurcation classification and hence the anatomical labeling was evaluated using cross-validation. One observer manually labeled the five BoI on each extracted tree. All the other bifurcations were assigned the label NI. Leaveone-out cross-validation was then run, where iteratively $K=30$ times, one case is used for testing while $K-1$ cases were selected to form the training set from which the bifurcation vectors, reference tree topology and its probability of appearance were learned. 
To evaluate the contribution of the regularizing prior term, we performed the above validation for three method variants: the first variant is a natural extension of [6] and is relying just on the SVM classification based on the bifurcation feature vectors with no regularization. The second variant has the regularization by topology added (SVM+TP), and can be considered as an adaptation of [5]. Finally, the third variant is the proposed one, which includes the prior term $(\mathrm{SVM}+\mathrm{TP}+\mathrm{AP})$, where the probability of appearance of a particular anatomical variability coming from the missing BoI is learned from the training set. The cross-validation results are presented in Table 1 .

Table 1. Performance of the three method variants. Scores for successfully labeling the complete vessel tree and detecting the individual bifurcations are given.

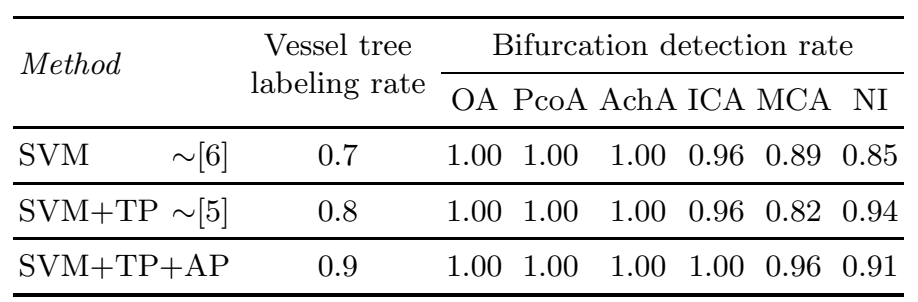

As seen from the first row, PcoA, AchA and OA bifurcations were well classified just based on their feature vectors. Gradually adding the regularization terms improved the tree labeling accuracy each time by $10 \%$. The proposed method (SVM+TP+AP) outperformed similar state of the art methods and had success rate of $90 \%$ for correctly labeling the complete vessel tree. The error occurred in three cases, when MCA bifurcation was detected as a NI and vice versa. A sample of correct anatomical labeling results is shown in Fig. 3 where the surface area of each corresponding vessel segment is labeled.

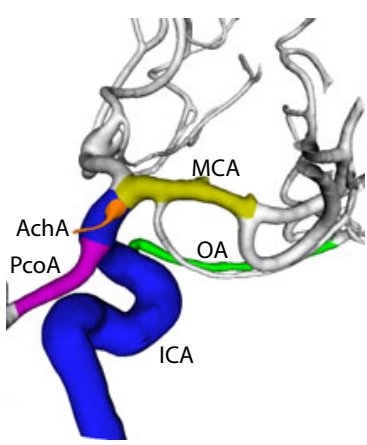

(a)

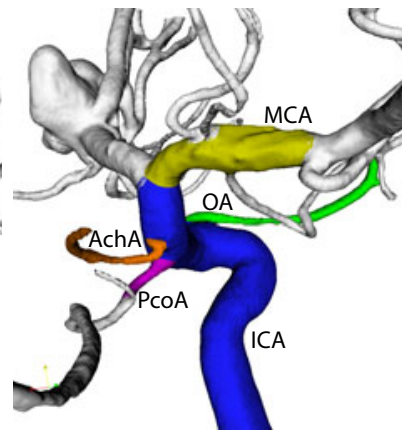

(b)

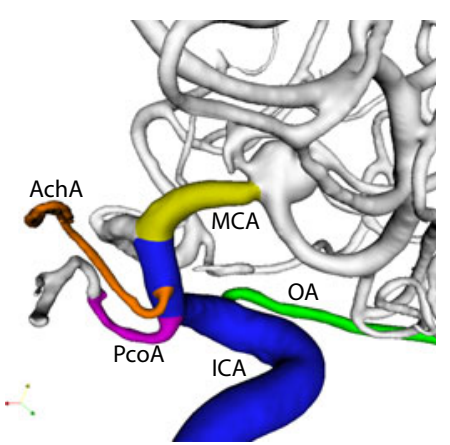

(c)

Fig. 3. Anatomically labeled vascular trees. Denoted vessels: ICA (blue), OA (green), PcoA (violet), AchA (orange), MCA (yellow) 
As the method is formulated in a probabilistic framework the candidate labelings can be ranked by their estimated probabilities. The method always selects the most probable one (the highest ranked one). However, in the case that selected labeling is visually observed to be incorrect, this enables us to select the next most probable candidate in the ranked list. For the three cases where the method assigned the incorrect labels, we searched for the correct labeling in the ranked list (Table 2). We can observe that the correct labeling appears as the second or at most the third most probable candidate.

Table 2. The three cases for which the labeling provided by the proposed method $(\mathrm{SVM}+\mathrm{TP}+\mathrm{AP})$ was incorrect. Position in the list of candidate labelings ranked by their probabilities is given for the selected and the correct labeling.

\begin{tabular}{clc}
\hline & \multicolumn{2}{l}{ position (probability) } \\
\cline { 2 - 3 } & selected & correct \\
\hline error case 1 & $1(0.61)$ & $2(0.20)$ \\
\hline error case 2 & $1(0.39)$ & $2(0.26)$ \\
\hline error case 3 & $1(0.36)$ & $3(0.15)$ \\
\hline
\end{tabular}

\section{Conclusion}

We presented a method for anatomical labeling of the main vessels forming anterior part of the Circle of Willis. From a set of prelabeled examples, the method is able to learn local bifurcation features as well as global variation in tree topology and their probabilities of occurrence. Focusing just on BoI makes it insensitive to the number of bifurcations that are extracted in between them. This is important as in general, total number of bifurcations and vessels largely varies between images as it heavily depends on the contrast and image resolution during acquisition as well as accuracy of segmentation method applied.

The current main limitation is that topologically correct candidates are obtained by computing maximal cliques of association graph, which is a NPcomplete problem. For our application, this was computationally feasible as in general trees could be pruned in a preprocessing step down to $N \sim 20$ prospective candidates. In the future, we plan to search for more efficient way of finding isomorphic mappings and also extend the anatomical labeling to the entire Circle of Willis.

Acknowledgments. This work has been partially funded by the CENIT programme of CDTI (cvREMOD CEN-20091044) and VPH-NoE project from FP7 of the European Union ( $\mathrm{nr}$ 223920). The work of H.B. is supported by the FI programme of AGAUR. The work of R.C. is supported by the Beatriu de Pinós, programme of AGAUR. A.F.F. is partially funded by the ICREA-Academia programme. 


\section{References}

1. Brisman, J.L., Song, J.K., Newell, D.W.: Cerebral aneurysms. N. Engl. J. Med. 355(9), 928-939 (2006)

2. Tschirren, J., McLennan, G., Palagyi, K., Hoffman, E.A., Sonka, M.: Matching and anatomical labeling of human airway tree. IEEE Trans. Med. Imag. 24(12), 1540-1547 (2005)

3. Mori, K., Hasegawa, J., Suenaga, Y., Toriwaki, J.: Automated anatomical labeling of the bronchial branch and its application to the virtual bronchoscopy system. IEEE Trans. Med. Imag. 19(2), 103-114 (2000)

4. Mori, K., Ota, S., Deguchi, D., Kitasaka, T., Suenaga, Y., Iwano, S., Hasegawa, Y., Takabatake, H., Mori, M., Natori, H.: Automated anatomical labeling of bronchial branches extracted from CT datasets based on machine learning and combination optimization and its application to bronchoscope guidance. In: Yang, G.Z., Hawkes, D., Rueckert, D., Noble, A., Taylor, C. (eds.) MICCAI 2009. LNCS, vol. 5762, pp. 707-714. Springer, Heidelberg (2009)

5. Mori, K., Oda, M., Egusa, T., Jiang, Z., Kitasaka, T., Fujiwara, M., Misawa, K.: Automated nomenclature of upper abdominal arteries for displaying anatomical names on virtual laparoscopic images. In: Liao, H., Edwards, E., Pan, X., Fan, Y., Yang, G.Z. (eds.) MIAR 2010. LNCS, vol. 6326, pp. 353-362. Springer, Heidelberg (2010)

6. Bogunovic, H., Pozo, J.M., Cardenes, R., Frangi, A.F.: Automatic identification of internal carotid artery from 3DRA images. In: Proc. IEEE EMBC, pp. 5343-5346. IEEE Press, Los Alamitos (2010)

7. Bogunovic, H., Pozo, J.M., Villa-Uriol, M.C., Majoie, C.B.L.M., van den Berg, R., Gratama van Andel, H.A.F., Macho, J.M., Blasco, J., San Roman, L., Frangi, A.F.: Automated segmentation of cerebral vasculature with aneurysms in 3DRA and TOF-MRA using geodesic active regions: An evaluation study. Med. Phys. 38, 210-222 (2011)

8. Cardenes, R., Bogunovic, H., Frangi, A.F.: Fast 3D centerline computation for tubular structures by front collapsing and fast marching. In: Proc. IEEE ICIP, pp. 4109-4112. IEEE Press, Los Alamitos (2010)

9. Antiga, L., Ene-Iordache, B., Remuzzi, A.: Computational geometry for patientspecific reconstruction and meshing of blood vessels from MR and CT angiography. IEEE Trans. Med. Imag. 22(5), 674-684 (2003)

10. Antiga, L., Steinman, D.A.: VMTK: the Vascular Modeling Toolkit (2011), http://www.vmtk.org

11. Antiga, L., Steinman, D.A.: Robust and objective decomposition and mapping of bifurcating vessels. IEEE Trans. Med. Imag. 23(6), 704-713 (2004)

12. Chang, C.C., Lin, C.J.: LIBSVM: a library for support vector machines (2011), http://www.csie.ntu.edu.tw/ cjlin/libsvm

13. Bron, C., Kerbosch, J.: Algorithm 457: finding all cliques of an undirected graph. Comm. ACM 16(9), 575-577 (1973) 